\title{
Experimental Measurement of a UAV Rotor's Acoustic Emission
}

\author{
Felix Lößle ${ }^{(凶)}$, Anna Kostek, Clemens Schwarz, and Rainer Schmid \\ German Aerospace Center (DLR), Institute of Aerodynamics and Flow Technology \\ (AS), Bunsenstraße 10, 37073 Göttingen, Germany \\ felix.loessle@dlr.de
}

\begin{abstract}
Besides aerodynamic performance, most unmanned aerial vehicles (UAVs) are limited by noise emission in their range of application. Especially in urban areas, the acceptance of UAV noise emission by the affected population is required. Therefore, one objective of DLR's project URBAN RESCUE is to investigate the noise emission and noise generation mechanisms of small rotors. In order to study noise emission, a test setup was established in DLR's $1 \mathrm{~m}$ wind tunnel. For different flight modes, acoustic measurements were conducted using a microphone array. For all test cases the noise emission of the rotor's blade passing frequency $(\mathrm{BPF})$ is higher in front of the rotor (upstream) than behind it. Due to higher relative velocities on the advancing side than on the retreating side, emission is asymmetric with respect to the flow direction. Additionally, a time-resolved background-oriented schlieren (BOS) setup was used to visualize tip vortices and study noise generation mechanisms.
\end{abstract}

Keywords: Noise emission - Small rotors - Acoustic measurements • Microphone array $\cdot$ Background-oriented schlieren

\section{Introduction}

Due to the complexity of a UAV rotor's flow dynamics, there is a high variety of tonal as well as broadband sound generation mechanisms. In many respects, noise emission mechanisms of small UAV rotors, helicopter rotors and propellers are similar. While helicopter rotors and propellers have been studied for quite long, UAV rotors have come to the fore in recent years. Therefore, most noise generation theories are based on experiments dealing with helicopter rotors and propellers.

Noise generation mechanisms can be classified into rotational noise, airfoil self-noise and interaction noise. Rotational noise can be subdivided into loading and thickness noise. The first emerges when rotating forces act on the blades' surface, whereas the second is caused by fluid displacement due to blade rotation. Considering the Ffowcs Williams-Hawkings (FWH) equation makes clear that loading noise exhibits a dipole and thickness noise a monopole character. Based on the FWH-equation Gutin [4] and later Hanson [5] derived a theoretical model for propeller rotational noise emission in time and frequency domains. Another approach is given by Succi [11], who derived an approximate solution for the

(C) The Author(s), under exclusive license to Springer Nature Switzerland AG 2021

A. Dillmann et al. (Eds.): STAB/DGLR Symposium 2020, NNFM 151, pp. 387-396, 2021.

https://doi.org/10.1007/978-3-030-79561-0_37 
FWH-equation for a rotating blade element in the time domain. Airfoil selfnoise occurs when boundary layer structures and near wakes interact with the airfoil. These noise sources contribute to rotor broadband noise. An overview of airfoil self-noise models is given in Brooks et al. [3]. Regarding interaction noise, blade vortex interaction (BVI) is of primary interest when helicopters are studied. When rotor blades interact parallelly with a tip vortex in forward flight or descent, high pressure fluctuations are induced on the leading edge resulting in strong acoustic pulses, which are rich in higher harmonics of the blade passing frequency $(\mathrm{BPF})[14]$.

In recent years, noise emission of small UAV rotor has been studied experimentally and numerically. Acoustic measurements with an isolated UAV rotor were performed with several microphone positions, for example by Tinney and Valdez [12] and Yang et al. [13]. Acoustic measurements were compared with numerical results by Sinibaldi and Marino [10] and Zawodny et al. [15]. However, noise emission and noise generation mechanisms of small UAV rotors are not as well examined and understood as those of helicopter rotors and propellers. So far a model of noise emission has only been derived for a rotor in hover [12]. In summary, a need for research on the field of small UAV rotor acoustics remains. One objective of DLR's project URBAN RESCUE is to gain a better understanding of noise emission and noise generation mechanisms of small UAV rotors. Furthermore, a comprehensive model for noise emission is going to be derived within the scope of the project. In this paper, a first experimental approach to investigate the properties of an isolated UAV rotor in hover and forward flight is presented. The analysis covers the operation of a UAV rotor at different tilt angles and varying wind tunnel velocities. Microphone measurements are conducted in a plane below the rotor (in the direction of typical observers). Spectra and directional characteristics for different flight modes are compared and the applicability of scaling laws established for helicopter rotors are validated for UAV rotors.

\section{Experimental Setup}

The test setup was established in DLR's $1 \mathrm{~m}$ tunnel in Göttingen. The object of investigation was a clockwise rotating "CAM-Carbon Light-Prop" manufactured by aero-naut Modellbau for multicopter applications with a diameter of $40.64 \mathrm{~cm}$ $\left(16^{\prime \prime}\right)$ and a pitch of $15.24 \mathrm{~cm}\left(6^{\prime \prime}\right)$. The test setup and a 3D-scan of the rotor is shown in Fig. 1. Due to space limitations, the rotor was installed vertically in the wind tunnel test section. In order to allow tilt angle variations from $-90^{\circ}$ to $25^{\circ}$ the rotor and its drive unit were mounted on a rotatable support system. A positive tilt angle represents a rearward-tilt of the rotor's tip path plane. In order to maintain the rotor's position when rotating, the rotor hub was located in the rotation axis of the rotatable support system (illustrated by the red line in Fig. 1 (a)). The wind tunnel's flow velocity was measured with a Prandtl probe in the nozzle outlet. All presented measurements were conducted with the rotor operating at a rotational speed of $4000 \mathrm{rpm}$ (corresponding to a hover tip speed 
of $84 \mathrm{~m} / \mathrm{s}$ ). Measurements with a load cell showed that with this settings the hovering rotor generates thrust of $13.6 \mathrm{~N}$. The nondimensional rotor advance ratio defined as

$$
\mu=\frac{V_{\infty}}{\Omega R}
$$

where $V_{\infty}$ is the magnitude of free-stream velocity, $\Omega$ the rotor's rotational frequency and $R$ the rotor radius is used to characterize the forward motion. The advance ratio varied within a range of 0 and 0.26 (corresponding to wind tunnel velocities between $0 \mathrm{~m} / \mathrm{s}$ and $22 \mathrm{~m} / \mathrm{s}$ ).

Acoustic measurements were performed in a plane below the rotor plane with a microphone array consisting of two lines of six Brüel \& Kjær 1/4" multi-field microphones (type 4961) each. The array was mounted on the wind tunnel's traverse in a plane parallel to the flow direction at a distance of $1140 \mathrm{~mm}$, which turned out to be sufficient to ensure non-affection of the microphones by the wind tunnel's shear layer. However, the exposure of microphones to the rotor's downwash couldn't be prevented for setups with tunnel velocities lower than $10 \mathrm{~m} / \mathrm{s}$. The measurement was carried out with 16 different array positions in order to cover an area of $1524 \mathrm{~mm} \times 1375 \mathrm{~mm}$ with increments of $100 \mathrm{~mm}$ in flow direction (x-axis) and $125 \mathrm{~mm}$ in the perpendicular direction (y-axis), as illustrated in Fig. 2. Each microphone's signal was calibrated with a Brüel \& Kjær pistonphone (type 4231) before and after the experiment. The wall and floor behind the microphone array was covered with acoustic foam to reduce reflections. The acoustic signals were sampled for $20 \mathrm{~s}$ at $50 \mathrm{kHz}$. The power spectral density (PSD) was calculated with Welch's method (20 non-overlapping Hann-windowed data segments of 50000 samples each).

In order to visualize the blade tip vortices of the rotor a time-resolved BOS system operated at $1.33 \mathrm{kHz}$ was used. A Phantom Veo 640L camera with a sensor consisting of $2560 \times 1600$ pixels was used and equipped with a $180 \mathrm{~mm}$ focal length lens at an aperture setting of $\mathrm{f} / 22$. The camera was placed at a distance of $3.26 \mathrm{~m}$ to the rotor hub and $6.31 \mathrm{~m}$ to the background. The background consisted of retroreflective foil printed with randomly placed dots and was illuminated by a high-power light-emitting diode (LED). The fundamentals of this measurement technique have previously been summarized by Raffel [9] and are not given here.

\section{Geometric Deviations of Directional Characteristics}

In order to get the rotor's directional characteristics, the acquired sound pressure level (SPL) of each microphone position was normalized to a distance of one meter relative to the rotor hub. Linear interpolation was carried out between grid points.

Directional characteristics calculated from measurement data should be carefully interpreted: First, acoustic waves are refracted passing the wind tunnel's shear layer. Second, there is atmospheric absorption when the pressure signal propagates. However, both effects are negligible due to low wind tunnel Mach numbers (up to 0.064) and small distances between shear layer and microphones [1]. 


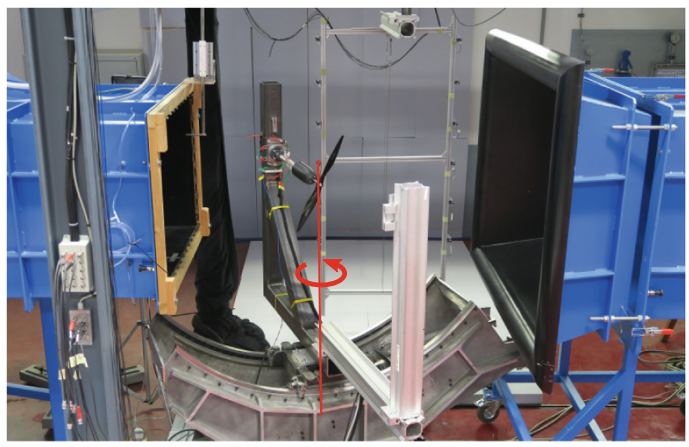

(a) Positioning of the rotor under investigation mounted on the rotatable support system in the wind tunnel; microphone array in the background

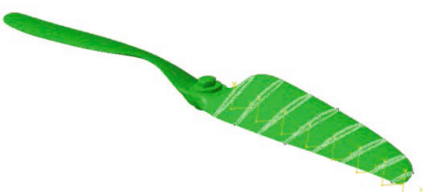

(b) Laser 3D-scanned geometry of the rotor of investigation

Fig. 1. Experimental setup of the acoustic rotor measurement in DLR's $1 \mathrm{~m}$ tunnel

Third, the array's distance to the rotor is not sufficient to allow measurements in the geometric far field (i.e. measuring distance is not much greater than the source region's extent). Hence the rotor's spatial extent causes two distorting effects on the noise hemisphere. On the one hand, if the effective acoustic source is not positioned in the rotor hub (as assumed for the hemisphere projection), the discrepancy between assumed and effective distance between source and microphones leads to SPL deviations. On the other hand, the rotor's spatial extent results in a deviation of the true emission angle. Both effects depend on the microphone position and can be estimated by geometric considerations. The maxima of deviations are expected if the acoustic source was located in the blade tips and are illustrated in Fig. 3. While the variation of SPL increases with elevation and reaches a maximum of $0.75 \mathrm{~dB}$, the deviation in emission angles peaks at elevations between $10^{\circ}$ and $20^{\circ}$ and doesn't exceed an absolute value of $10^{\circ}$.

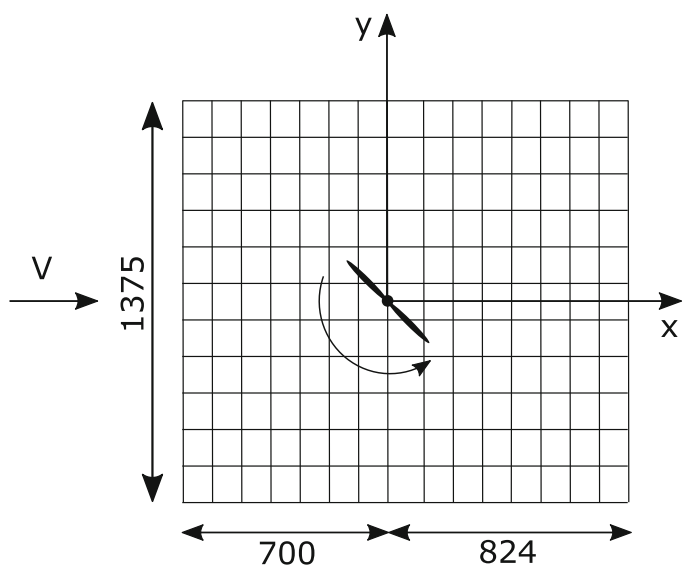

Fig. 2. Grid of microphone positions (view on the rotor's suction side) 


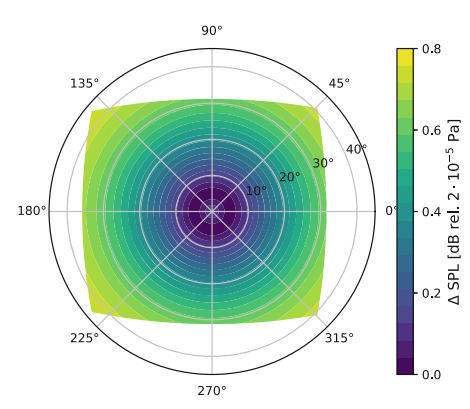

(a) Maximum SPL deviation

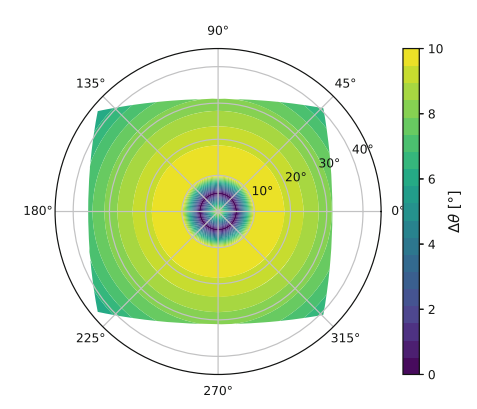

(b) Maximum angular distortion

Fig. 3. Deviations due to the source region's extent, if measurements take place in the rotor's geometric near field

\section{Results and Discussion}

\section{Results of Acoustic Measurements for a Single Observer}

In this section, the spectra of a single microphone located in front of the rotor are considered $(x=-700 \mathrm{~mm}, y=62.5 \mathrm{~mm})$. An overview about spectra at advance ratios $\mu$ between 0.05 and 0.26 and tilt angles $\alpha$ between $-10^{\circ}$ and $10^{\circ}$ is given in Fig. 4. Two spectra at an advance ratio $\mu=0.19$ are presented in Fig. 5 in more detail. All spectra show tones at the BPF and its harmonics as well as broadband noise. In the following, the characteristics of these three types of noise are discussed separately.

First of all, the BPF is considered. The spectra show that the SPL of the BPF exceeds the level of higher harmonics by more than $10 \mathrm{~dB}$ and therefore determines the overall sound pressure level (OASPL). Figure 6 shows the influence of advance ratio $\mu$ and tilt angle $\alpha$ on the SPL of the first harmonic. (The SPL of the first harmonic was obtained by integrating the PSD in the frequency range of $\mathrm{BPF} \pm 10 \mathrm{~Hz}$.) The dependence of SPL on advance ratio $\mu$ is theoretically predicted: Due to small blade volume, loading noise is expected to be the dominant noise generation mechanism [6]. Since loading noise has dipole character, sound pressure follows a sixth-power law scaling with Mach number [7], which has been experimentally confirmed by Aravamudan et al. [2]. Moreover, scaling of forces with Mach number must be taken into account for rigid rotors. Considering this effect, Lowson and Ollerhead found an eighth power law for rotor rotational noise [7]. Based on this theory, noise is mainly generated at fast moving blade areas, in particular at the blade tips on the advancing side. Using the tip Mach number of the advancing side, the blue and red line in Fig. 6 represent the sixth and eighth power law. The eighth power law captures the rise of the first harmonic's SPL at advance ratios higher than 0.1 well, but underpredicts the increase for smaller advance ratios. 


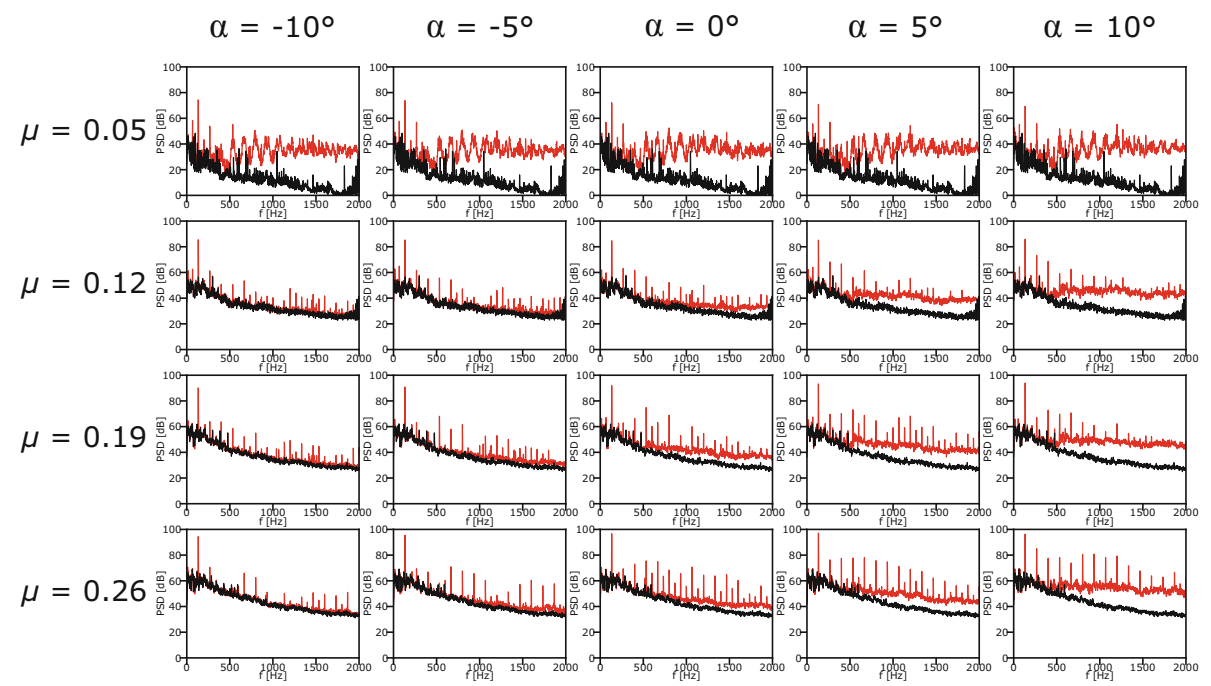

Fig. 4. Spectra of rotor noise at several advance ratios $\mu$ and tilt angles $\alpha$ at position $x=-700 \mathrm{~mm}, y=62.5 \mathrm{~mm}$, the acoustic signal of the rotor is indicated by the red lines, background noise by the black lines

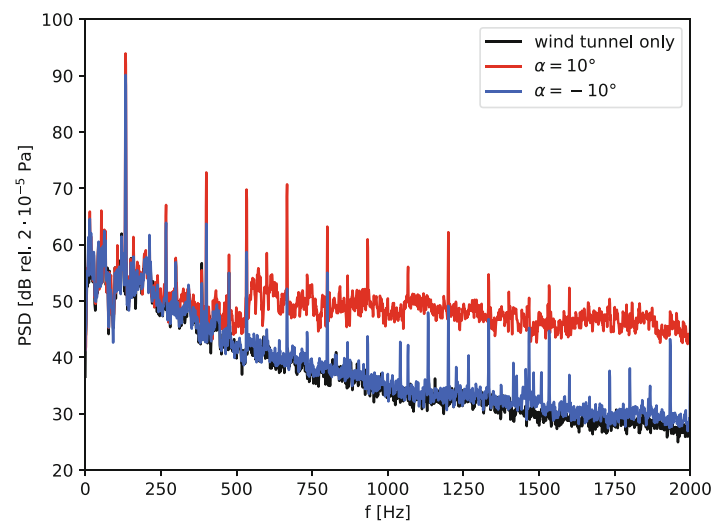

Fig. 5. Measured PSD at position $x=-700 \mathrm{~mm}, y=62.5 \mathrm{~mm}$, the rotor was operated at an advance ratio $\mu=0.19$

Finally, the characteristics of higher harmonics and broadband noise are addressed. The SPL of the higher harmonics grows with increasing advance ratio and with increasing tilt angle. The level of broadband noise emission is dependent on the rotor's tilt angle. At negative angles, the rotor's broadband noise is drowned out by the wind tunnel and therefore can't be studied. However, at tilt angles of $0^{\circ}$ and higher, the rotor's broadband noise is sufficiently loud to be distinguishable from the environmental noise for frequencies greater than $500 \mathrm{~Hz}$. 


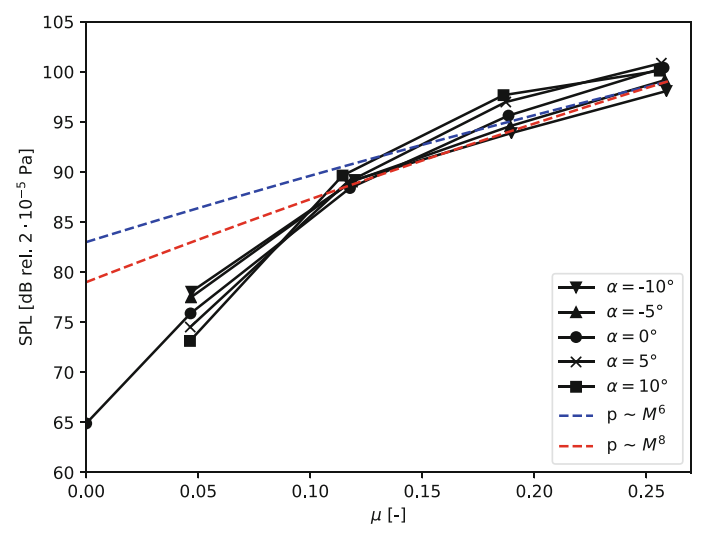

Fig. 6. SPL of the rotor's first harmonic at several advance ratios $\mu$ and tilt angles $\alpha$ at position $x=-700 \mathrm{~mm}, y=62.5 \mathrm{~mm}$

Two aerodynamic effects explain the dependence of the raise of higher harmonics and broadband noise on the advance ratio and tilt angle mentioned before. On the one hand, flow separation occurs in an intensified way at higher advance ratios and tilt angles. Flow separation mainly influences broadband noise emission [3]. On the other hand, the interaction of rotor blades with tip vortices constitutes an additional noise mechanism. Measurements performed on a model helicopter showed that BVI results in the increase of higher harmonics [8]. In order to discover whether it comes to an interaction of rotor blades and tip vortices, tip vortices were visualized with background-oriented schlieren (BOS). The results at a tilt angle of $0^{\circ}$ and three different advance ratios are illustrated in Fig. 7. In hover and slow forward flight tip vortices are uniformly convected downstream. On the contrary, the convection is found unsteady at advance ratios $\mu=0.12$ and higher. Due to flat angles of convection the vortex system remains in the vicinity of the rotor for longer, increasing the probability of interaction with rotor blades.

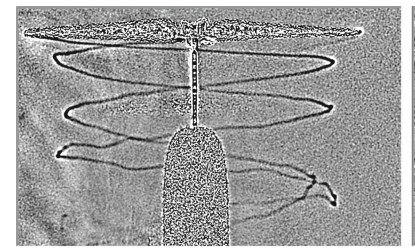

(a) $\mu=0$

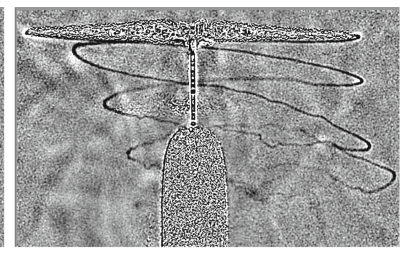

(b) $\mu=0.05$

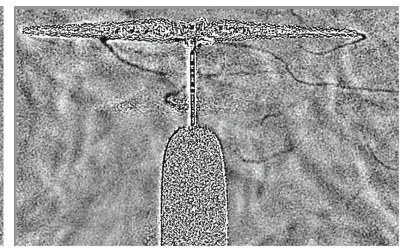

(c) $\mu=0.12$

Fig. 7. Visualization of tip vortices at three different advance ratios $\mu$ using BOS 


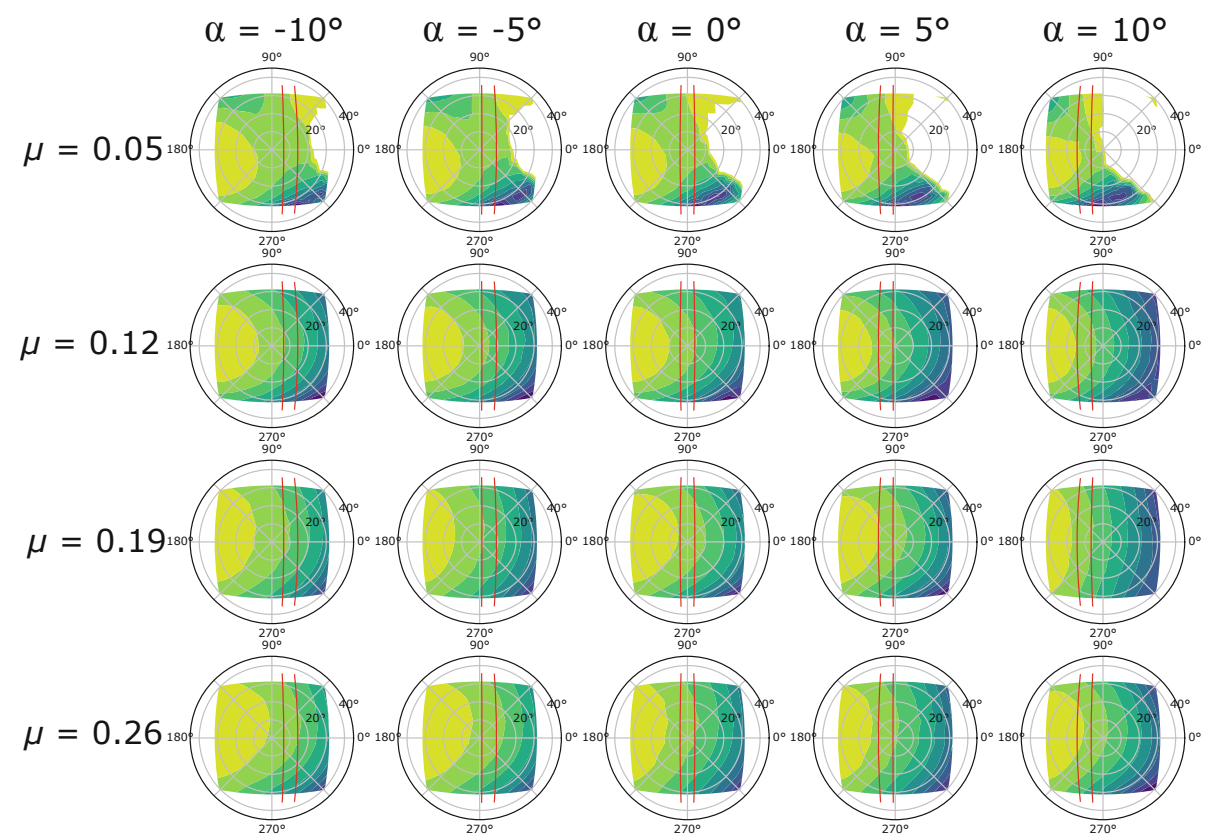

Fig. 8. Measured noise emission spheres of the rotor's first harmonic at several advance ratios $\mu$ and tilt angles $\alpha$, elevation is in radial and azimuth in circumferential direction plotted

\section{Directional Characteristics}

As mentioned before, the first harmonic determines the rotor's OASPL in all test cases. Thus, the directional characteristic of only the rotor's first harmonic is considered in the following. In Fig. 8, SPL spheres at advance ratios $\mu$ between 0.05 and 0.26 and tilt angles $\alpha$ between $-10^{\circ}$ and $10^{\circ}$ are given. One test case SPL sphere is illustrated in more detail in Fig. 9. At an advance ratio $\mu=0.05$, the rotor's downwash influences the microphone array, which leads to areas in the rotor's rear where the microphones are saturated. These areas are masked in Fig. 8 (blank areas). The color themes of the individual hemispheres are independent from one another and correspond to the minimum and maximum within the individual test cases. The vertical red lines represent the contour of the rotor support structure projected onto the hemisphere. Apparently, the SPL sphere of the rotor's first harmonic is not noticeably influenced by the suspension's shadowing due to its small width relative to wave length. For all test cases, the noise emission of the rotor is higher in front of the rotor (upstream i.e. flight direction) than behind it. Moreover, emission is asymmetric in relation to the inflow direction. At an advance ratio $\mu=0.05$, noise emission is slightly stronger on the rotor's retreating side than on its advancing side. However, with increasing advance ratio noise emission shifts from the retreating side to the advancing side. At an advance ratio $\mu=0.12$, the directional characteristic is nearly 


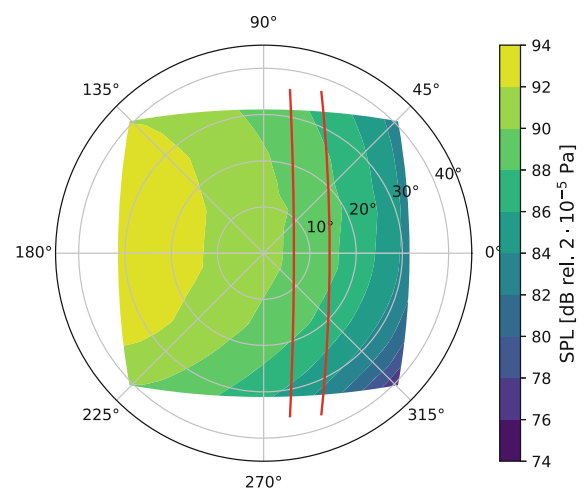

Fig. 9. Measured noise emission sphere of the rotor's first harmonic, the rotor was operated at an advance ratio of $\mu=0.19$ and a tilt angle of $\alpha=-10^{\circ}$, elevation is in radial and azimuth in circumferential direction plotted

symmetric. Higher advance ratios result in emission levels which are higher on the advancing side than on the retreating side. As mentioned above, the spanwise asymmetry in the emission directivity can be traced back to relative velocities which are higher on the advancing than on the retreating rotor side. Future work will focus on a better understanding of emission effects causing the directivity.

\section{Conclusion}

An acoustic test setup for small rotors was established in DLR's 1m tunnel, using a microphone array for measurement. The results show that the rotor's first harmonic determines the OASPL for all test cases and depends on the advance ratio. At advance ratios higher than 0.1 a correlation was found between measurement and Lowson and Ollerhead's eighth power scaling law (in reference to the tip Mach number of the advancing side). For higher harmonics and broadband noise an increase of SPL with growing advance ratio and rotor tilt angle can be observed. We consider the onset of flow separation and interactions of rotor blades with tip vortices to be responsible. The visualization of tip vortices using BOS for a tilt angle of $0^{\circ}$ indicates that interaction occures at an advance ratio of 0.12 and higher. The directivity of the first harmonic is asymmetric. At low advance ratios the noise emission is slightly stronger on the rotor's retreating than on its advancing side. With increasing advance ratio the higher noise emissions shift from the retreating side to the advancing side.

For further experiments it's advisible to use an aeroacoustic wind tunnel with anechoic character, if available. In order to exclude the possibility of a significant impact of the room's acoustic properties on the directional characteristics, measurements will also be conducted with a counterrotating rotor. In order to derive a comprehensive model for noise emission, experiments combining acoustic and load measurements are planned. Furthermore, additional experiments with 
rotors of varying size, number of blades and pitch angles expands the model' scope of application. Future work will also focus on assessing the sound emission effects in more detail, including localizing their exact sources and characterizing the underlying flow effects using beamforming.

\section{References}

1. Amiet, R.K.: Refraction of sound by a shear layer. J. Sound Vib. 58(4), 467-482 (1978)

2. Aravamudan, K.S., Lee, A., Harris, W.L.: A simplified Mach number scaling law for helicopter rotor noise. J. Sound Vib. 57(4), 555-570 (1978)

3. Brooks, T.F., Pope, D.S., Marcolini, M.A.: Airfoil Self-noise and Prediction. NASA Reference Publication 1218 (1989)

4. Gutin, L.: On the sound field of a rotating propeller. Translated in NACA TM $1195(1936)$

5. Hanson, D.B.: Helicoidal surface theory for harmonic noise of propellers in the far field. AIAA J. 18(10), 1213-1220 (1980)

6. Hanson, D.B.: Influence of propeller design parameters on far-fild harmonic noise in forward flight. AIAA J. 18(11), 1313-1319 (1980)

7. Lowson, M.V., Ollerhead, J.B.: Studies of helicopter rotor noise. USAAVLABS Technical report 68-60 (1969)

8. Martin, R.M., Hardin, J.C.: Spectral characteristics of rotor blade/vortex interaction noise. J. Aircr. 25(1) (1988)

9. Raffel, M.: Background-oriented schlieren (BOS) techniques. Exp. Fluids 56(3), 1-17 (2015). https://doi.org/10.1007/s00348-015-1927-5

10. Sinibaldi, G., Marino, L.: Experimental analysis on the noise of propellers for small UAV. Appl. Acoust. 74(1), 79-88 (2013)

11. Succi, G.P.: Design of quiet efficient propellers. In: SAE Business Aircraft Meeting, Society of Automotive Engineers Paper SAE79-0584, pp. 2039-2052 (1979)

12. Tinney, C.E., Valdez, J.: Acoustic scaling for small rotors in hover. In: AHS International 75th Annual Forum, Philadelphia (2019)

13. Yang, Y., Liu, Y., Li, Y., Arcondoulis, E., Wang, Y., Li, W., Huang, B.: Aerodynamic and aeroacoustic characteristics of a multicopter propeller during forward flight. In: AIAA Propulsion and Energy Forum, Cincinnati (2018)

14. Yu, Y.H.: Rotor blade-vortex interaction noise. Prog. Aerosp. Sci. 36, 97-115 (2000)

15. Zawodny, N.S., Boyd, D.D., Burley, C.L.: Acoustic characterization and prediction of representative, small-scale rotary-wing unmanned aircraft system component. In: American Helicopter Society 72nd Annual Forum, West Palm Beach (2016) 